\title{
Thyroid Screening in Obstetric Patients
}

\author{
Dr. B. Srinivas Rao ${ }^{1}$, Dr. E. Indira ${ }^{2}$, Dr. R.Rama ${ }^{3}$, \\ Assistant Professors,Dept Of OBG, Siddartha Medical College, Dr.NTRUHS, Vijayawada, Andhra Pradesh.
}

\begin{abstract}
Background: Although gestational hyperthyroidism is uncommon (0.2\%), Hypothyroidism occurs in $2.5 \%$ to $3 \%$ of women and is predictive of reduced neonatal and child neuropsychological development and maternal obstetric complications. Postpartum thyroid dysfunction occurs in 5-9\% of women and is associated with anti thyroid peroxidase antibodies in 10\% of women in early pregnancy. Therefore, screening of thyroid dysfunction in pregnancy should be considered. T4 and TSH measurements could be used to screen for hypothyroidism, which would require levothyroxine treatment. T4 supply is crucial for maturation of fetal nervous system.

Methods: Study was done in 1000 pregnant women. Screening was done by estimating T3, T4, TSH.

Results: Out of 1000 pregnant women, 30 were diagnosed as hypothyroidism.

Among them 5 were diagnosed as overt hypothyroidism and 25 cases were sub clinical hypothyroidism. 18 cases were diagnosed as hyperthyroidism. Among them 2 were overt hyperthyroidism and 16 were sub clinical hyperthyroidism. These patients are followed up and hypothyroid patients were treated with levothyroxine, hyperthyroid patients were treated with Propylthiouracil.

Conclusion: Potential and inadequately treated hypothyroid patients present with problems in pregnancy. While adequately treated, hypothyroid or hyperthyroid women get normal ongoing pregnancy. So to identify these potential or overt hypothyroid women, and risk based screening has been shown to miss at least $30 \%$ of cases. So universal thyroid screening with T3, T4, TSH must be done during prenatal, at first booking, and repeated at 8 weeks interval thereafter in pregnancy.
\end{abstract}

Keywords: Hypothyroidism, Hyperthyroidism, T3, T4, TSH, Levothyroxine, Propyl thyouracil.

\section{Introduction}

Thyroid disorders are one of the common endocrinological problems in women. They are 10 times more common in women than in men. They are relatively more common in women of reproductive age group, so they are frequently encountered during pregnancy. Incidence of hypothyroidism in pregnancy is $2.5-3 \%$ and incidence of hyperthyroidism in pregnancy is $0.05-1.7 \%$. There are many adverse affects of thyroid dysfunction on pregnancy in the antepartum, intrapartum, postpartum period and also there is increased perinatal morbidity and mortality, fetal and neonatal thyroid dysfunction.

Diagnosis of thyroid dysfunction is difficult during pregnancy because, physiological changes occurring during pregnancy may mimic symptoms of hypo/hyperthyroidism ie tiredness, constipation, weight gain, anemia, carpal tunnel syndrome, thinning of hair which are features of hypothyroid, also occur in normal pregnancy. Anxiety, heat intolerance, sweating, warm extremities, amenorrhea seen in hyperthyroidism also occur in normal pregnancy. We should screen pregnant women with a suspicion to diagnose hypo/ hyperthyroidism. We depend on biochemical measurement of thyroid hormones to diagnose hypo/hyperthyroidism. They must be interpreted according to pregnancy specific changes because changes occur in thyroid physiology during pregnancy.

\section{Aims \& objectives}

1. To screen thyroid disorders among obstetric patients.

2. To study the effect of thyroid disorders on pregnancy \& perinatal outcome.

3. To study the effect of pregnancy on thyroid disorders.

\section{Patients and Methods}

Study was conducted at Dept of OBG, Govt General Hospital, Vijayawada for a period of TWO years from November 2014 to October 2016. All the pregnant women who attended the Antenatal OPD, who are willing for the study were included. Patients with Autoimmune disease \& Endocrinological diseases and not willing patients were excluded. 


\section{Review of Literature \\ Biochemical Assesment of Thyroid Funtion In Pregnancy.}

Thyroid binding globulin increase, total T4 increase, circulating FT4, FT3 levels are largely unchanged. $1^{\text {st }}$ trimester : TSH decreases, T4 increases. $2^{\text {nd }}$ trimester: TSH, T4 remain in normal limits. $3^{\text {rd }}$ trimester: TSH increases, FT4 decreases. This biochemical hypothyroidism is well tolerated with no clinical features of hypothyroidism as euthyroidism is maintained by an increase sensitivity of tissues to thyroid hormones. It could be due to physiological adaptation to try to conserve energy in preparation for parturition and may be due to increase fetal demand. In pregnancy FT4 and TSH should be monitored rather than total T4 and total T3.

\section{Anatomy of thyroid gland. 1,2,3}

Thyroid gland is one of the largest endocrine glands of the body and weighs about 15-20 gms in adults. Thyroid gland is shaped like a "Butterfly" having 2 lateral lobes connected by a central isthmus $1,2,3$. It is composed of globular follicles, which are filled with an amber colored sticky colloid. The follicles are lined by thyroid follicular cells, which are either cuboidal or squamous epithelial cells and are involved in secreting thyroglobulin and along with the attached iodine atom, form the colloid, which acts as the precursor of the thyroid hormones. Another type of endocrine cells found in the follicular epithelium are called parafollicular cells which are involved in the production of calcitonin 2,3.

\section{Functions of the Thyroid Gland.4}

The absorption of the iodine is one of the main functions of the thyroid gland, as the thyroid cells are identified as the only cells in the body, which can absorb iodine.4. While the thyroid gland is involved in the synthesis and secretion of two important hormones T3 (Tri-iodothyronine) and T4 (Tetra-iodothyronine) play crucial role in metabolic homeostasis of the body. Para follicular cells of the thyroid gland synthesize calcitonin, a hormone related in calcium homeostasis.

\section{Regulation of the Thyroid Hormone Synthesis}

Maintenance of normal metabolic activity of the body is made possible by coordinated cycle of interactions between the hypothalamus, pituitary gland and thyroid gland. Regulation of thyroid hormone synthesis through these interactions is a classical example of endocrine feed back mechanism.1,2. Production of TSH is under the control of TRH, produced by the hypothalamus, which acts as positive regulator of TSH synthesis and secretion.

\section{Thyroid Hormone Mode of Action.}

The major metabolic hormones after being carried through transport proteins, thyroid hormones enters the cells either positively or through monocarboxylate transporter 8 (MCT8).In the cytoplasm most of the T4 is deiodinated to form T3, which enters the nucleus. Two types of receptors are present $\alpha$ and $\beta$.

\section{Blood Test 5,6.}

Thyroid function tests are performed to check the functional status of the thyroid. A number of tests are available for testing the function of the thyroid these include total T3, T4, free T3,T4 and serum TSH level assay. TRH assay checks the pituitary function. Measurement of thyroid hormone binding proteins such as TBG, TTR, pre albumin and albumin are some of the tests done, though not routine on clinical diagnosis. In addition the measurement of thyroid hormone precursor protein thyroglobulin is another assay to measure thyroid function. Thyroid specific auto antibodies such as TSH receptor antibody TRAb, thyroid peroxidase antibody (TPOAb) and thyroglobulin antibody (TGAb).

\section{Other Tests 7,8.}

Other tests involved in laboratory diagnosis of thyroid dysfunction include Ultrasonography, radioactive uptake, thyroid scanning and fine needle aspiration cytology (FNAC).

\section{Thyroid in Pregnancy 9, 10, 11, 12, 13, 14.}

Thyroid physiology plays a major role in pregnancy. It has been reported thyroid diseases occurs in about 1$2 \%$ of pregnant women and are more in women of child bearing age, during pregnancy as well as in postpartum 9,10 . It is well known that thyroid diseases affect the outcome of pregnancy. Since the developing fetus synthesizes thyroid hormone only by first trimester, it depends on maternal thyroid hormones for organogenesis and CNS development as well as its general growth. Moreover, thyroid hormones are essential for the maintenance as well as successful completion of normal pregnancy 11,12. Even after the delivery thyroid hormones are essential during the postpartum period, playing significant role in improving lactation. However 
the early detection of thyroid dysfunction and treatment of mother during gestation improve the outcome. 13 . Half life of TBG is also reported to increase as a result of altered glycosylation by estrogen during gestation 13. Iodine deficiency may cause thyroid goitre in pregnant women .14. Estrogen levels increase during pregnancy this is associated with increase in concentration of TBG in serum. Estrogen causes altered glycosylation of TBG, leading to increased half life of TBG 9,10. HCG plays crucial role in thyroid physiology during pregnancy, elevated HCG is associated with increased T3 and T4 causes hyperemisis.

\section{Role of Iodine During Pregnancy And Postpartum 15,16,17,18.}

During pregnancy, there is increased loss of urinary iodine due to increased GFR 15. Deficiency of Iodine can cause reduced maternal T4, leading to maternal goitre in iodine deficient area, placental abruption and preeclampsia. It can also lead to neurological abnormality, low birth weight and loss of fetus 16 . WHO recommends a daily dietary intake of $250 \mu \mathrm{g}$ of iodine during lactation and pregnancy with a maximum limit up to $500 \mu \mathrm{g} /$ day 17. Iodine is in the form of iodised salt is suggested. However, excessive iodine intake may result in thyrotoxicosis, aggravation of auto immune goitre and increased suppression of thyroid gland .18.

\section{Transplacental Transport of Thyroid Hormone 19,20.}

Transplacental transport of thyroid hormones is highly significant for the fetus since it depends on maternal hormone until it produces its own 19. Placenta produces thyroid hormone transport protein and transthyronine during the early and later periods of gestation. These transport proteins plays a crucial role in transplacental transport of thyroid hormones. The placenta can transport small amount of T3 and T4 .20. Transplacental transport of T4 is regulated by placental deiodinase type 3, while fetus depends on placental deiodinase type 2 for T3 conversion from T4. 20. Transthyritin doesn't carry T3. Placenta also involved in iodine transport to the fetus, the supporting fetal production of thyroid hormones.

\section{Fetal Thyroid Physiology}

Fetal thyroid develops from 5 weeks of pregnancy begins function from 10 - 12 weeks, till that time it depends on maternal T4. Later fetus starts synthesizing T3 \&T4 independent from the mother. Placenta is impermeable to TSH. T3,T4 transfer occurs in $1^{\text {st }}$ trimester, later becomes impermeable. Iodides, thioamide, releasing hormones, thyroid stimulating immunoglobulin cross only early through placenta.

\section{At Parturition}

Acute rise in TSH with corresponding increase in T3,T4 levels occurs in the fetus peaking at 24-36 hours.

\section{Discussion}

Incidence of hypothyroidism is 3-5\%.Thyroid disorders are one of the most common endocrine disorders in women during pregnancy. sahu et.al in 2009 reported the incidence of sub clinical and overt hypothyroidism in India as $6.5 \%$ and $4.6 \%$ respectively.

\section{Effect of Pregnancy on Hypothyroidism 21.}

There is need for increase in the dose of thyroxine during pregnancy.

\section{Pregnacy outcome in Hypothyroidism}

Maternal: There increase incidence of Miscarraige, PIH, anaemia, fetal growth restriction, Placental abruption, PPH, Cardiac dysfunction. Rare complications of hypothyroidism was OHSS.

Fetal: Congenital abnormality, Still birth, Prematurity, Increased risk of fetal distress in labor, Neonatal Encephalopathy, Reduced Intellect. 22.

\section{Diagnosis of Hypothyroidism in Pregnancy}

Tiredness, constipation, weight gain, anaemia, carpal tunnel syndrome, thinning of hair is seen during pregnancy also. we need to rely on biochemical measurement for diagnosis.

1. Check serum TSH level as soon as pregnancy is confirmed.

2. For newly diagnosed hypothyroid women, initial levothyroxine dosage is based on severity of hypothyroidism. For overt Hypothyroidism administer $1.5-2 \mathrm{mcg} / \mathrm{kg} / \mathrm{day}(1.6 \mathrm{mcg} / \mathrm{kg} / \mathrm{day})$. The therapy can be started with initial dose of $0.10 \mathrm{mg} / \mathrm{day}$ and increased by $0.025-0.05 \mathrm{mg}$ increments at $2-4$ weeks interval depending upon the TSH levels.

3. For previously diagnosed women monitor serum TSH every 3-4 weeks during first half of pregnancy and every 6 weeks thereafter.

4. Adjust levothyroxine dosage to maintain serum TSH $<2.5 \mathrm{mU} / \mathrm{L}$ 
5. After delivery reduce levothyroxine to pre pregnacy dosage, and check serum TSH in 6 weeks and adjust levothyroxine dose.

\section{Diagnosis of Neonatal Hypothyrodism 23.}

Ideally serum TSH and T3 are estimated from an elute of whole blood collected on filter paper by a heel prick between $4^{\text {th }}$ and $6^{\text {th }}$ days of life. If the screening blood TSH is more than $40 \mathrm{mU} / \mathrm{L}$ then the child is called for retesting but treatment is not indicated unless rising levels are documented.

\section{Management of Hypothyroidism 24.}

According to American association of clinical endocrinologists (AACE), although routine serum thyrotrophic testing during early pregnancy is recommended. Serum TSH analysis is indicated in all pregnant women with goitre, high antithyroid antibody, family history of thyroid disease, any history of auto immune endocrine disease, symptoms suggestive of hypothyroidism. Thyroxine therapy is mainstay of medical treatment of hypothyroidism in pregnancy. Women already on levothyroxine therapy before pregnancy should undergo thyrotrophic testing and dosage should be adjusted. Thyroxine is considered to be safe in pregnancy and lactation. Thyroid hormone should be measured in each trimester when thyroid disease is stable and women clinically and biochemically euthyroid.

\section{Hyperthyroidism In Pregnancy 25,26,27.}

2 in 1000 pregnancies present with overt hypothyroidism and $1.7 \%$ of pregnancies with mild or sub clinical hyperthyroidism. Most of women generally experience the symptoms before pregnancy. A few may have them during the course of pregnancy for the first time, the common causes are Grave's disease, subacute thyroiditis, toxic multinodular goitre, Toxic adenoma, TSH-dependent thyrotoxicosis, exogenous T4 or T3, Iodine induced hyperthyroidism, and HCG associated transient thyrotoxicosis.

\section{Effects of Pregnancy on Hyperthyroidism 28.}

Pregnancy does not seem to influence the long term course of hyperthyroidism. What ever be the aetiology, Grave's disease remits in 2nd and 3rd trimester resulting in a reduced requirement for anti thyroid medication. In first trimester and puerperium, flare of Graves disease occurs because immune state in 2nd and 3rd trimester cause decrease in thyrotropin receptor stimulating antibodies (TRSab).In first trimester decrease absorption of drug occurs, increase in HCG levels. In puerperium TRSab increases.

\section{Outcome of Pregnancy Complicated By Hyperthyroidism.}

If hyperthyroidism is well controlled before and throughout pregnancy, outcome for the mother and baby are generally good.

If poorly controlled

Maternal complications: Preeclampsia, Preterm labor, Abruptioplacenta, Congestive heart failure, thyroid storm.

Fetal complications: Still births, IUGR, Oligohydramnios, Abortions, Increased perinatal mortality.

\section{Transient Hyperthyroidism 29.}

Hyperemesis gravidarum and gestational trophoblastic disease are two pregnancy specific conditions that may be causative in the development of hyperthyroidism. These conditions require immediate identification and treatment.

\section{Postpartum Thyroiditis.}

Transient autoimmune thyroiditis is consistently found in $5-10 \%$ of women during the first year after delivery.

\section{Thyroid Nodules in Pregnancy.}

A Thyroid nodule is a lesion within the thyroid gland that is discrete in nature, palpable and can be differentiated from the surrounding thyroid parenchyma in an ultrasonography. To ascertain the presence of thyroid nodules during pregnancy thyroid function tests are warranted.

\section{Thyroid Storm.}

It is very rare complicates 1-2\% of thyroid toxic pregnancies. It is an acute exacerbation of thyrotoxicosis in patients with preexisting hyperthyroidism. Maternal mortality is up to $20 \%$ is noticed with this condition. There is often a precipitating factor like surgery, administration of RAI, infection, labour, delivery, anaesthesia, abrupt withdrawal of ATD. 


\section{Neonatal Screening 30.}

Fetal screening is essential in mothers with thyrotoxicosis. It is done to check fetal growth, heart rate and fetal neck and to rule out thyrotoxicosis that may present with fetal tachycardia. Hyperthyroidism in the mother treated with PTU have low T4, rise in TSH in the neonate leading to transient hypothyroisism.

\section{Complications of Fetal Hyperthyroidism.}

Intellectual impairement, hydrops fetalis, intrauterine death, polyhydramnios, malposition due to fetal goitre causing oesophagial obstruction and neck extension, neonatal hyperthyroidism risk is $1 \%$. Half life of thyroid stimulating hormone antibody is 21 days, where as half life of PTU is less than 7 days. So the manifestations of hyperthyroidism may not become apparent until 7-10 days.

\section{Results}

In our study out of 1000 pregnant women, 30 were diagnosed as hypothyroidism, among them 5 (16\%) were diagnosed as overt hypothyroidism and $25(84 \%)$ cases were sub clinical hypothyroidism. 18 cases were diagnosed as hyperthyroidism, among them $2(11 \%)$ were overt hyperthyroidism and $16(89 \%)$ were sub clinical hyperthyroidism.

In our study complications of hypothyroidism-20\% of patients are present with mild PIH,13.3\% aneamia,10\% IUGR,10\% Preterm delivery, 10\% IUD,3.3\% severe PIH,33.3\% had normal pregnancy. Lowell E et al (1988) studied 28 hypothyroidism cases at parkland hospital Maternal complications were anemia 31\%, Preeclampsia 44\%, Abruption 19\%, PPH 19\% and cardiac dysfunction. Low birth weight 31\%, fetal death $12 \%$ in overt hypothyroidism.

In our study complications of hyperthyroidism-PIH 33.3\%, Oligohydramnios $22.2 \%$ IUGR $22.2 \%$, Normal pregnancy $22.2 \%$.Buckshee K et al. 1994 studied 32 pregnancies complicated by hyperthyroidism preterm labour $25 \%$, PIH $22 \%$, thyroid crisis $9 \%$, IUGR $13 \%$.

Hypothyroid \& Hyperthyroid Cases in Pregnanacy

\begin{tabular}{|l|l|l|}
\hline TYPE OF THYROID & NUMBER OF CASES & PERCENTAGE \\
\hline OVERT HYPOTHYROIDISM & 5 & $16 \%$ \\
\hline SUBCLINICAL HYPOTHYROIDISM & 25 & $84 \%$ \\
\hline OVERT HYPERTHYROIDISM & 2 & $11 \%$ \\
\hline SUBCLINICAL HYPERTHYROIDISM & 16 & $89 \%$ \\
\hline
\end{tabular}

Hypothyroidism \& Hyperthyroidism in Pregnancy - Age Group

\begin{tabular}{|l|l|l|}
\hline Age In Years & Number Of Cases & Percentage $(\%)$ \\
\hline $15-20$ Years & 02,04 & 7,22 \\
\hline $21-25$ Years & 20,10 & 67,55 \\
\hline $26-30$ Years & 07,04 & 23,23 \\
\hline 31-35 Years & $01,--$ & $03,--$ \\
\hline
\end{tabular}

Diagnosed Cases Of Hypothyroidism \& Hyperthyroidism

\begin{tabular}{|l|l|l|}
\hline DIAGNOSIS AT & NUMBER OF CASES & PERCENTAGE $(\%)$ \\
\hline PREGNANCY & 25,10 & $83.33,55$ \\
\hline POST PREGNANCY & 5,04 & $16.66,23$ \\
\hline
\end{tabular}

Parity in Hypothyroidism \&Hyperthyroidism

\begin{tabular}{|l|l|l|}
\hline Parity & Number Of Cases & Percentage(\%) \\
\hline Primigravida & 15,5 & 50,28 \\
\hline Gravida -2 & 07,5 & 23,28 \\
\hline Gravida -3 & 05,8 & 17,44 \\
\hline Gravida -4 & $03,--$ & $10,--$ \\
\hline
\end{tabular}

Complications Of Hypothyroidism \&Hyperthyroidism In Pregnancy

\begin{tabular}{|l|l|l|}
\hline Complications & Number Of Cases & Percentage $(\%)$ \\
\hline Mild PIH & 6,6 & $20,33.33$ \\
\hline Severe PIH & $1,--$ & $3.3,--$ \\
\hline IUGR & 3,4 & $10,22.22$ \\
\hline Oligohydramnios &,-- 4 &,-- 22.22 \\
\hline Preterm Delivery & $3,--$ & $10,--$ \\
\hline Anaemia & $4,--$ & $13.33,--$ \\
\hline IUD & $3,--$ & $10,--$ \\
\hline
\end{tabular}




\begin{tabular}{|l|l|l|}
\hline Normal & 10,4 & $33.33,22.22$ \\
\hline
\end{tabular}

Neonatal Birth Weights in Hypothyroidism \& Hyperthyroidism

\begin{tabular}{|l|l|l|}
\hline Birth Weight In Kgs & Number of Neonates & Percentage $(\%)$ \\
\hline $1.5-2 \mathrm{Kg}$ & 06,03 & 20,17 \\
\hline $2-2.5 \mathrm{Kg}$ & 15,12 & 50,67 \\
\hline $3-3.5 \mathrm{Kg}$ & 09,03 & 30,16 \\
\hline
\end{tabular}

APGAR Score in Hypothyroidism \& Hyperthyroidism in Neonates

\begin{tabular}{|l|l|l|}
\hline APGAR Score & Number of Neonates & Percentage(\%) \\
\hline$<7$ & 10,4 & 33,23 \\
\hline$>7$ & 20,14 & 67,77 \\
\hline
\end{tabular}

\section{Conclusion}

Maintaining of thyroid hormone levels within the gestational normal range is very important during gestation both for successful completion of pregnancy as well as for appropriate fetal development. Physiological changes during pregnancy modify thyroid function. There is a need for maintaining TSH levels at specific range gestation. Hypothyroidism may cause complications in both mother and fetus. Diagnostic techniques for evaluating hypothyroidism include test for thyroid and antithyroid antibodies includes T3, T4, TSH, TSHR, anti TPO and TABG. Hypothyroid pregnant women to be treated with thyroxin and adequate iodine intake in case of iodine deficiency. Grave's disease is one of the most common cause of hyperthyroidism in pregnancy. Hyperemesis gravidarum and gestational trophoblastic disease are two pregnancy specific conditions that may develop hyperthyroidism. Treatment of Grave's disease is with propylthiouracil. In order to prevent maternal, neonatal complications, screening of all pregnant women irrespective of risk factors should be done.

\section{Summary}

Hypothyroidism if well controlled does not pose a major problem in pregnancy except for adjustment of thyroxin dose and increased incidence of pregnancy induced hypertension. Dose of thyroxin if increased, it stresses the necessity for monitoring the thyroid function tests during pregnancy. In hyperthyroid pregnant women there is an increased incidence of hypertension complicating pregnancy and also increased risk of neonatal hyperthyroidism. Hence neonatal thyroid function needs to be tested and treated accordingly. Pregnancy with thyroid disorder should be treated as a high risk pregnancy and managed appropriately by both obstetrician and endocrinologist.

\section{Bibliography}

[1]. Guyton C,Hall j.Thyroid metabolic hormones.Textbook of medical physiology 11 th ed,New Delhi:Elsevier,Reed Elsevier India Private Ltd:2007:931-943

[2]. Jameson J,Weetman A.Disorders of thyroid gland .In:Fauci A,BraunwaldE,kasper D,Hauser S,Longo D,Jameson J,et al;eds Harrison's principles of Internal medicine $17^{\text {th }}$ ed USA.

[3]. Marieb E,Hoehn K. The Endocrine system Human anatomy and physiology $.7^{\text {th }}$ ED San Francisco,CA;Pearson Education Inc;2007:620-625.

[4]. The function of thyroid gland.Learning info .http://health.learning info.org/function thyroid gland.htm.Accessed March 10,11 A..D.

[5]. Joshi S Laboratory evaluation of thyroid function JAPI.2011;59(supplement):14-20.

[6]. SI units of clinical data .University of North Carolina:https://www. Unc.edu. Rowlett/units/scales/clincaldata.html. Accessed March 3.11 A.D.

[7]. Blum M .Ultrasonography of Thyroid function .Thyroid manager.1-41:http://www.thyroid manager.org/chapter6c/chapter6c frameh.html. Accessed march 12011.

[8]. Thyroid function tests NIH.http://www.endocrine.niddk.nih. gov/pubs/thyroidtests/thyroidtests. Pdf. Accessed March 3,2011.

[9]. Fitzpatrick DL ,Russell MA. Diagnosis and management of thyroid disease in pregnanacy .Obstet Gynecol Clin North Am.2010;37(2):173-193

[10]. Galofre JC ,Davies TF .Autoimmune thyroid in pregnancy .:A review .J womens health (Larchmt).2009;18(11):1847-1856.

[11]. Choski NY, Jahnke GD, stHc,Shelby M.Role of thyroid hormones in human and laboratory animal reproductive health. Birth defects Res B Dev Reprod Toxicol.2003;68(6)479-491.

[12]. ReidS.Interventions for clinical and subclinical hypothyroidism in pregnancy (Review).wley.http://onelinelibrary.wiley.com/o/Cochrane/clsysrev/articles/CD007752/pdf_fs.html. Accesed march , 2001.

[13]. Lazarus J. Thyroid functions in pregnancy. British medical bulletin.2010;1-12

[14]. Thyroid disease and pregnancy. American thyroid association. http://www.thyroid.org/patients/brouchers/Thyroid Dis pregnancy broch.pdf. Accessed March1,2011.15. management of thyroid dysfunctions during pregnancy and postpartum. The endocrine society.htttp://www.endo.society.org/guidelines/final/upload /clinical guideline-mangament-of-thyroid-dysfunction-duringpregnancy-postpartum.pdf. Accesed march3,2011.

[15]. Banerjee S. thyroid disorders in pregnancy .JAPI.2011;59 (supplement) :32-34.

[16]. Varez-PedereroIM, Guxens M, Mendez M, et al.Iodine levels and thyroid hormones in healthy pregnant women and birth weight of their offspring.Eur JEndirinol.2009; 160(3):423-429. 
[17]. Reaching Optimal Iodine Nutrition in pregnant and lactating women and Young Children.WHO. http://www.who.int/nutrition/publications/WHOstatment_IDD pregnancy. pdf.Accessed march3,2011.

[18]. Marwaha R,Gopalakrishnan S.Facts of iodine supplementation.JAPI. 2011;59 (supplement):7-10

[19]. Landers KA.McKinnon BD,LiH,Subramaniam VN, Mortimer RH, Richard K.Carrier-mediated thyroid hormone transport into placenta by placental transthyretin.J Clin Endocrinol Metab.2009;94(7):2610-2616

[20]. Negro R, Schwatz A, Gismondi R, Tinelli A, Mangieri T, stangnaro-Green A.Increased pregnancy loss rate in thyroid antibody negative women with TSH levels between 2.5 and 5.0 in the first trimester of pregnancy. JClin Endocrinol Metab.2010;95(9):E44E48.

[21]. Galofre JC, Davies TF. Autoimmune thyroid disease in pregnancy: a review . J Womens Health (larchmt).2009 18(11);1847-1856.

[22]. Haddow JE. Palmaki GE. Allen WC, et al. Maternal thyroid deficiency during pregnancy and subsequent neuropsychological development of the child. N Engl J Med.1999-341(8):549-555.

[23]. Berghout A, Wiersinga W. Thyroid size and thyroid function during pregnancy : an analysis. Eur.J.endocrinol. 1998: 138(5): 536542

[24]. Gharib H, Papini E, Paschke R et al. American Association of clinical Endocrionologist, Associazione Medici Endocrinologi, and European Thyroid Association Medical Guidelines for Clinical Practice for the Diagnosis and Management of Thyroid nodules. Endocr Preact.2010;16 Suppl 1:1-43.

[25]. Benerjee S.Thyroid disorders in pregnancy. Supplement to JAPL.2011;59

[26]. Fantz CR,gogo-Jack S, Ladenson JG, Gronowski AM. Thyroid function during pregnancy. Clin Chem.1999;45(12):2250-2258

[27]. Galfre J,Davies T. Autoimmune thyroid disease in pregnancy; a review J.Womens Health (Larchmt).2009;18(11):1847-1856.

[28]. Zainuddin Z, Shaker AAH. Hyperthyroidism in pregnancy. The Family physician.2005;13(3).

[29]. Jueckstock JK, Kaestner R, Mylonas I. Managing hyperemesis gravidarum: a mutimodal challenge. BMC Med.2010;8:46.

[30]. Newborn Screening For Sick or Preterm Newborns. Utah Department of Health. 